\title{
ANÁLISE DA FUNCIONALIDADE DE PACIENTES IDOSOS INTERNADOS EM UM HOSPITAL ESCOLA
}

Maitê Silva Vicente dos Santos; Pontifícia Universidade Católica do Rio Grande do Sul - Programa de Residência Multiprofissional em Saúde do Idoso PREMUS-HSL; maite.vicente1@hotmail.com

Renata Beckenkamp Krause; Pontifícia Universidade Católica do Rio Grande do Sul - Programa de Residência Multiprofissional em Saúde do Idoso PREMUS-HSL; renatak968@gmail.com

Elisiane Vidal; Hospital São Lucas da PUCRS; elisianevidal@hotmail.com

Flavia Franz; Pontifícia Universidade Católica do Rio Grande do Sul; Serviço de Fisioterapia HSL/PUCRS; flavia.franz@pucrs.br

Clarissa Netto Blattner; Pontifícia Universidade Católica do Rio Grande do Sul; Programa de Residência Multiprofissional em Saúde do Idoso PREMUS-HSL; clarissa.blattner@pucrs.br

\section{RESUMO}

Introdução: A pandemia causada por um novo tipo de coronavírus, COVID-19, ameaça os idosos que são mais suscetíveis a desenvolver sintomas graves e cursar com necessidade de hospitalização, que são naturalmente prolongadas e contribuem com a perda de funcionalidade. Objetivo: Avaliar mobilidade e funcionalidade de pacientes internados em um hospital geral até sua alta hospitalar, identificando o tempo e desfecho da internação. Métodos: Trata-se de um estudo de coorte prospectivo, realizado em um hospital em Porto Alegre, entre maio e junho de 2020. Este trabalho obteve a aprovação do Comitê de Ética em Pesquisa da Pontifícia Universidade Católica do Rio Grande do Sul. Um relatório foi gerado através do prontuário eletrônico, onde foram filtradas as hospitalizações em unidade de internação de pacientes com 60 anos ou mais e diagnóstico de COVID-19, com prescrição de fisioterapia. Foram analisados o Escore de Perme do primeiro e último atendimento de fisioterapia, tempo e desfecho da internação. Resultados: O relatório apresentou 15 indivíduos, onde 3 foram excluídos por falta de dados registrados. A média de idade da amostra foi de 69 anos ( \pm 60 - 83). A média de tempo de internação foi de 33,5 $( \pm 11)$ e do Escore de Perme na primeira avaliação foi de 9 e na segunda avaliação 8,5 pontos. Quanto ao desfecho, 4 indivíduos receberam alta, 2 foram transferidos e 6 foram a óbito. Conclusão: Concluímos que os idosos desta pesquisa apresentaram uma alta taxa de mortalidade, tempo de internação prolongado e discreta redução de funcionalidade durante período da internação.

Palavras-chave: Funcionalidade; Assistência a idosos; Fisioterapia. 